\title{
COVID-19, racism, and the pursuit of health care and research worthy of trust
}

\author{
Lisa A. Cooper ${ }^{1,2,3,4}$ and Deidra C. Crews ${ }^{1,2,4}$ \\ Johns Hopkins Center for Health Equity, Johns Hopkins University, ${ }^{2}$ Department of Medicine, Johns Hopkins University School of Medicine, ${ }^{3}$ Department of Health, Behavior, and Society, Johns Hopkins \\ Bloomberg School of Public Health, and ${ }^{4}$ Welch Center for Prevention, Epidemiology, and Clinical Research, Johns Hopkins University, Baltimore, Maryland, USA.
}

B y February 2020, the US had reported its first deaths from COVID-19. It was not long before the overrepresentation of African American, Latino, and Native American populations among reported coronavirus infections and deaths became apparent. African Americans and Latino individuals have been approximately three times as likely to become infected and twice as likely to die from the virus as Whites. At the same time, calls for racial justice have erupted in the wake of several high-profile killings of African Americans - most notably, the murder of George Floyd on May 25, 2020, witnessed by the world on video recording, after more than eight minutes of having a police officer kneel on his neck as he repeatedly cried out, "I can't breathe."

\section{Acknowledging the legacy of structural racism}

COVID-19 disparities and the reactions of many Americans to racially motivated violence are not surprising. The social contract concept suggests that individuals consent to yielding some of their freedoms and submitting to authority in exchange for protection of their remaining rights or maintenance of the social order (1). A level of trust in authority is inherit in this consent. However, over the course of history, African Americans and other people of color have been excluded from the social contract made by the US government with its citizens or subjected to the repercussions of a broken contract. These breeches of contract are reflected in structural racism. In the US, structural or institutional racism includes policies and structures that allow the dominant group (White Americans) to differential- ly allocate desirable opportunities and resources to people of color, subjecting them to harm and failing to protect them (2). Policies inherent to institutional racism, such as residential segregation, have long-lasting adverse health effects on the population. Residential segregation places people of color in neighborhoods that lack social investment, leading to less access to high-quality health care and education, healthful foods, well-paying jobs, and safe housing, physical, and social environments (3).

In health care, structural racism is evident in the well-documented disparate access to care and the quality of health care received by people of color in the US. These disparities are pervasive across settings, populations, health conditions, and levels and types of care. Researchers have pointed to factors that contribute at multiple levels - from policies and community resources to organizational attributes and clinician behaviors. It is not surprising, then, that African Americans, Latino individuals, and those in other racial/ ethnic minority groups report lower levels of trust in physicians and health care systems than do Whites (4).

In research, structural racism shows up in the disproportionately low levels of funding for health disparities research (5), the lack of diversity among leadership and investigators in the research enterprise, the underrepresentation of racial and ethnic minorities in research studies, and the lack of engagement of communities of color in the design and conduct of research studies, including the dissemination of results. For example, African Americans make up 13\% of the US population, but only $5 \%$ of patients enrolled in clinical trials that

Conflict of interest: The authors have declared that no conflict of interest exists.

support US Food and Drug Administration approval of new drugs (6).

\section{Restoring trust in health care and research}

Physicians and researchers can draw lessons from two interrelated approaches to reducing racism and enhancing trustworthiness in health care and biomedical research: relationship-centered care (RCC) and structural competence (Figure 1). RCC can be defined as care in which all participants appreciate the importance of their relationships with one another, recognizing that all healing occurs within relationships (7). In medical practice, patient-physician relationships provide the context for exchanging information, arriving at diagnoses, choosing treatments, and assessing the outcomes of care. Patient-physician relationships are central in RCC, but there are also other important relationships to consider, including the relationships of clinicians with themselves (i.e., self-awareness), with colleagues (reflected in the relational climates of our institutions), and with their communities (which include potential partners and participants for research studies) (8). Relationship dimensions linked to positive outcomes include communication, partnership, respect, knowing, trust, and concordance (shared social identities and values) (8). Unfortunately, numerous studies have shown that these dimensions are present at lower levels in the relationships between ethnic minority patients and their physicians, ethnic minority health professionals and their colleagues, and communities of color and the researchers who study them (8).

Because disparities in health care and research also have their roots in structures and policies that are broader than interpersonal relationships, structural competence has been proposed as another approach to addressing the myriad 


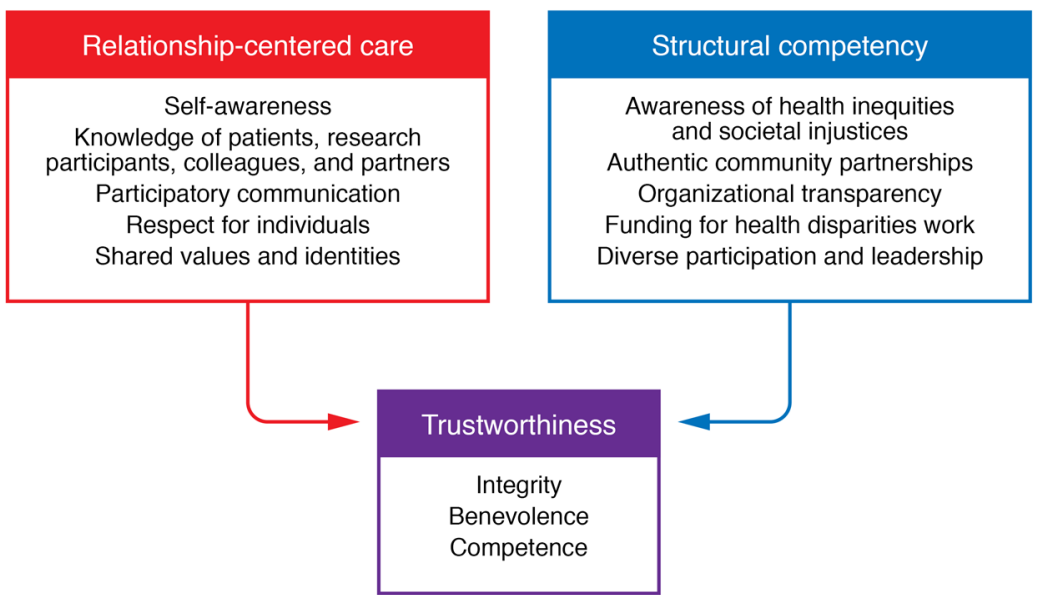

Figure 1. Approaches to reducing racism and enhancing trustworthiness in health care and biomedical research. RCC values the opinions and needs of all parties involved in care and includes self-awareness of clinicians; knowledge of patients, research participants, colleagues, and partners; engaged, participatory communication; mutual respect; and shared values and identities. Addressing structural competency requires an awareness of inequities, community partnerships, transparency, increased funding for health disparities work, and health care and research workforce, participation, and leadership that reflect the diversity of the population. Together, these approaches can help improve trust in health care and biomedical research.

factors leading to poor health in communities of color (9). The health care system needs new emphasis on ensuring access to high-quality care and preventive health care, addressing patients' broader social needs as part of health care delivery, diversifying the health care workforce to more closely reflect the demographic composition of the patient population, and providing competency-based curricula on structural factors, including structural racism (10). These lessons can also be applied to biomedical research. Efforts should be made to more effectively address barriers to the engagement of patients from communities of color in research, to diversify the biomedical research workforce, and to provide greater funding and infrastructure for research addressing health disparities.

\section{Implementing an actionable plan}

How can we practically apply lessons from RCC and structural competence to achieve health care delivery and biomedical research that are equitable and worthy of the trust of communities of color? Beginning with the clinician or clinician-scientist relationship to self, successful approaches include training in self-awareness and professionalism, such as employing the race Implicit Association Test with opportunities for self-reflection, perspective taking, and enhancing emotional regulation skills (10).

To improve our relationships with patients and research participants, we can use relationship-centered behaviors (9), such as probing their perspectives with open-ended questions, asking for their input on decisions, listening respectfully, showing empathy and support for their emotional needs, and avoiding racially biased treatment or stereotyping behaviors when interacting with them. RCC also means providing patients and research participants with information in short, clear statements with opportunities for questions and preparing them for what they should expect from diagnostic tests, treatments, and research protocols. Although more evidence is needed, many of these behaviors have been associated with higher levels of trust by patients and research participants (11).

To mitigate health disparities, health professional education should include training to promote structural competency. This training should include developing knowledge regarding how health inequities are driven by forces at institutional and societal levels and providing the necessary skills to take these factors into account when caring for patients and interacting with research participants. With regard to relationships with colleagues, organizational research suggests several best practices, including ensuring diversity of organizational members and leaders, valuing and using varied ways of thinking for problem solving, mindfulness (self-awareness, openness to new ideas), mutual respect, and trustworthiness (the ability to be relied upon based on benevolence, integrity, and competence) (12, 13). Health systems should demonstrate commitment to socially disadvantaged groups through authentic partnerships with trusted agents; provide clear, timely, and accurate information using contextually appropriate methods; monitor and reward the reduction of disparities in care; and increase clinician time and support for rapport building and continuity of care with socially complex patients (14).

Community-based participatory research (CBPR) principles and practices are highly instructive for relationships with communities (15). CBPR approaches include bringing community members into research as partners, not just subjects; using community members' lived experiences to understand health problems and design activities that improve health care; connecting community members directly with how the research is done; and providing timely research results to participating communities. Further research is needed to determine metrics of success in community engagement between health systems and communities; however, several interpersonal and organizational practices lead to improved academic and workplace environments, organizational excellence, health care quality, shorter lengths of stay, and improved patient outcomes $(12,16)$.

\section{Bringing about change}

As clinicians and researchers, the best place we can begin is where we are. We can use our spheres of influence to take individual, institutional, and professional group actions to bring about positive change. As individuals, we can commit to becoming more aware of our biases, living in accordance with our values, and upholding the principles of our profession. We can commit to becoming more relationship centered in our interactions with patients, research participants, colleagues, and community partners. We can hold our institutional leaders accountable for implementing policies that will make 
our workplaces more diverse, inclusive, respectful, and collaborative. We can help our professional organizations to advocate effectively with policymakers to provide greater funding and support for health care, research, and services that benefit the socially disadvantaged by making opportunities more equitable and communities healthier.

These actions are critical as we navigate the challenges of preventing the spread of COVID-19 through public health education and practice, diagnosing and caring for patients, and conducting research to identify safe and effective vaccines and treatments. In taking these steps, we will be doing our part to ensure that everyone in our society benefits - especially our patients, research participants, colleagues, and communities experiencing racism and health inequities - and that we are worthy of their trust.

Address correspondence to: Lisa A. Cooper, 2024 East Monument Street, Suite 2-500, Baltimore, Maryland 21287, USA. Phone: 410.614.3659; Email: lisa.cooper@jhmi.edu.
1. Williams DL. Rousseau's Social Contract:An Introduction. Cambridge University Press; 2014.

2. Williams DR, Lawrence JA, Davis BA, Vu C. Understanding how discrimination can affect health. Health Serv Res. 2019;54 Suppl 2:1374-1388.

3. Bailey ZD, Krieger N, Agenor M, Graves J, Linos $\mathrm{N}$, Bassett MT. Structural racism and health inequities in the USA: evidence and interventions. Lancet. 2017;389(10077):1453-1463.

4. Schwei RJ, Kadunc K, Nguyen AL, Jacobs EA. Impact of sociodemographic factors and previous interactions with the health care system on institutional trust in three racial/ethnic groups. Patient Educ Couns. 2014;96(3):333-338.

5. Hoppe TA, et al. Topic choice contributes to the lower rate of NIH awards to African American/black scientists. Sci Adv. 2019;5(10):eaaw7238.

6. Berger JS, et al. Reporting and representation of race/ethnicity in published randomized trials. Am Heart J. 2009;158(5):742-747.

7. Beach MC, Inui T, Relationship-Centered Care Research Network. Relationship-centered care. A constructive reframing. J Gen Intern Med. 2006;21 Suppl 1:S3-S8.

8. Cooper LA, Beach MC, Johnson RL, Inui TS. Delving below the surface. Understanding how race and ethnicity influence relationships in health care. JGen Intern Med. 2006;21 Suppl 1:S21-S27.

9. Murphy KA, Ellison-Barnes A, Johnson EN, Cooper LA. The clinical examination and socially at-risk populations: the examination matters for health disparities. Med Clin North Am. 2018;102(3):521-532.

10. Williams DR, Cooper LA. Reducing racial inequities in health: using what we already know to take action. Int J Environ Res Public Health. 2019;16(4):E606.

11. Martin KD, Roter DL, Beach MC, Carson KA, Cooper LA. Physician communication behaviors and trust among black and white patients with hypertension. Med Care. 2013;51(2):151-157.

12. Safran DG, Miller W, Beckman H. Organizational dimensions of relationship-centered care. Theory, evidence, and practice. J Gen Intern Med. 2006;21 Suppl 1:S9-15.

13. Schnackenberg AK, Tomlinson EC. Organizational transparency: A new perspective on managing trust in organization-stakeholder relationships. Journal of Management. 2016;42(7): 1784-810.

14. Wesson DE, Lucey CR, Cooper LA. Building trust in health systems to eliminate health disparities. JAMA. 2019;322(2):111-112.

15. Israel BA, et al. Community-based participatory research: a capacity-building approach for policy advocacy aimed at eliminating health disparities. Am J Public Health. 2010;100(11):2094-2102.

16. Drahota A, et al. Community-academic partnerships: a systematic review of the state of the literature and recommendations for future research. Milbank Q. 2016;94(1):163-214. 